\title{
Bending crystals. Solid state photomechanical properties of transition metal complexes containing semiquinonate ligands
}

\author{
CORTLANDT G PIERPONT \\ Department of Chemistry and Biochemistry, University of Colorado, \\ Boulder, Colorado 80309, USA \\ e-mail: Pierpont@Colorado.edu
}

\begin{abstract}
The properties of transition metal complexes containing catecholate and radical semiquinonate ligands have often been found to be unusual and unexpected. Crystals of $\mathrm{Rh}(\mathrm{CO})_{2}$ (3,6-DBSQ), containing the 3,6-di-tert-butyl-1,2-semiquinonate ligand, form as long thin needles that are observed to bend reversibly upon irradiation with NIR light. Crystallographic characterization reveals a stacked solid state lattice with planar molecules aligned with metal atoms atop one another. Electronic spectra recorded in the solid state and in solution show an intense band at $1600 \mathrm{~nm}$ that maps the energy dependence of crystal bend angle. The transition is a property of the stacked assembly, rather than of an individual complex molecule, and appears associated with an MLCT process that transfers charge from an antibonding band formed by interacting $\mathrm{Rh} d_{z}^{2}$ orbitals to the vacant quinone $\pi^{*}$ orbital. Related observations have been made on the $\left[\mathrm{Co}(\mu \text {-pyz)(3,6-DBSQ) }(3,6-\mathrm{DBCat})]_{n}\right.$ polymer. Photomechanical properties appear associated with electronic transitions that lead to a physical change in axial length of a linear polymer, coupled with a soft solid state lattice that permits axial contraction/expansion without crystal fracture.
\end{abstract}

Keywords. Semiquinonate; transition metal; charge transfer; photomechanical property.

\section{Introduction}

Prior to the early 1970's there were many reports on transition metal complexes formed with catecholate ligands, mainly in the international literature on analytical chemistry, but few well-characterized complexes, and no reports on complexes characterized structurally ${ }^{1}$. Over the past 25 years studies on the coordination chemistry of catecholate and semiquinone ligands have defined a significant new direction in inorganic chemistry ${ }^{2}$. Some of the observations on complexes of these ligands have been surprising and unexpected. These include the emergence of the radical semiquinonate form of quinone ligands as a common electronic state in coordination chemistry ${ }^{3}$, and observations on facile metal-quinone electron transfer in simple complexes of redox active transition metal ions ${ }^{4}$. In this review we describe novel observations on the photomechanical properties of quinone complexes in the solid state.

\section{Photomechanical property of $\operatorname{Rh}(\mathrm{CO})_{2}(3,6-\mathrm{DBSQ})$}

Many of the important features of quinone complexes are derived from a similarity in energy between metal-valence and quinone $\pi^{*}$ orbitals. This property is responsible for intense, low-energy charge transfer transitions that often extend well into the infrared in 
energy. In the course of studies on quinone complexes of rhodium, crystals of $\mathrm{Rh}(\mathrm{CO})_{2}$ (3,6-DBSQ) (3,6-DBSQ - 3,6-di-tert-butyl-1,2-semiquinonate) were observed to bend reversibly when irradiated with light from a tungsten lamp ${ }^{5}$. A simple experimental arrangement was used to investigate light response (figure 1).

Crystals of $\mathrm{Rh}(\mathrm{CO})_{2}(3,6-\mathrm{DBSQ})$ form as long thin needles. A small reflective surface glued to the tip of a crystal mounted along the length of the needle axis has been used to monitor crystal bend angle. Angular measurements have been made by observing the displacement of a reflected laser beam on a screen. The incident light source is the tungsten lamp from a film projector plugged into a variac for intensity control. When the incident light source is switched on, the crystal bends and the laser beam is deflected on the screen. The power from the variac must be increased slowly to avoid breaking the crystal; crystals that have thickness appropriate for X-ray characterization break rather than bend. Angle is dependent upon crystal thickness and light intensity; thin crystals show greatest flexibility with angles of roughly $45^{\circ}$. Experiments carried out to investigate the energy sensitivity of bend angle have shown that angle-dependence maps the contour of an optical transition centered at approximately $1600 \mathrm{~nm}$ (figure 2). The

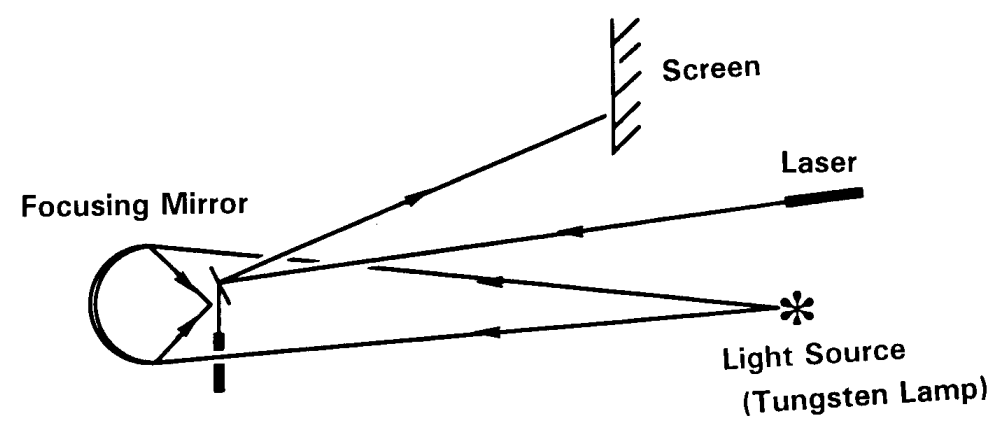

Figure 1. Schematic of experimental arrangement to study light response.

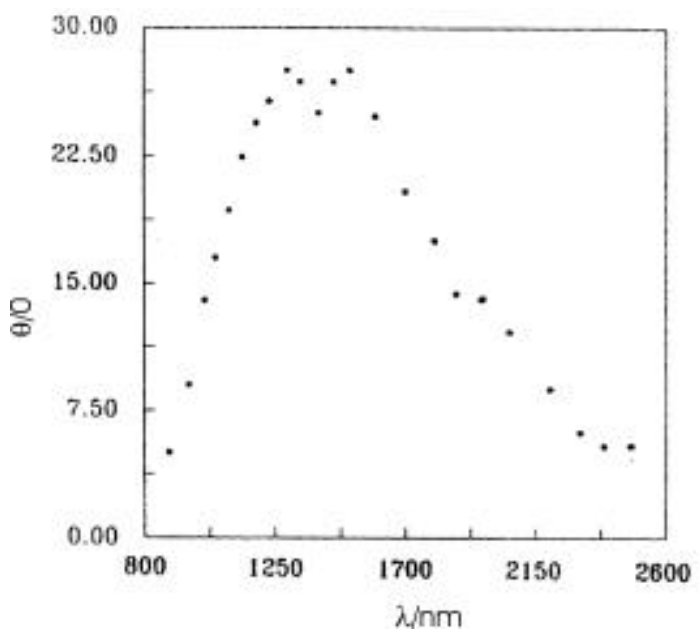

Figure 2. Plot of crystal bend angle vs wavelength of incident light. 
solid-state electronic spectrum of $\mathrm{Rh}(\mathrm{CO})_{2}(3,6-\mathrm{DBSQ})$ recorded as a dilute $\mathrm{KBr}$ disk is shown in figure 3 . The most prominent feature of the spectrum is an intense band in the $1600 \mathrm{~nm}$ region that maps the plot of bend-angle dependence on wavelength. A second feature of lower intensity appears near $800 \mathrm{~nm}$. This band is associated with a decomposition product that forms upon exposure of the complex to aerobic dioxygen. The nature of this product is not known, but crystals that have been exposed to air for the period of a day or more fail to bend due to the formation of this decomposition product on the surface.

A crystal of $\mathrm{Rh}(\mathrm{CO})_{2}(3,6-\mathrm{DBSQ})$ has been used to determine the solid-state and molecular structure of the complex. As expected, the geometry about the Rh center is square planar (figure 4). The molecular packing arrangement in the crystal consists of columns of complex molecules, stacked along the long needle axis of the crystal, with the $\mathrm{Rh}$ atoms of adjacent planar units aligned atop one another (figure 5). Inequivalent $\mathrm{Rh}-$ $\mathrm{Rh}$ separations within the stack are 3.252(4) and 3.304 (5) $\AA$, well beyond the length of a $\mathrm{Rh}-\mathrm{Rh}$ bond.

Electronic spectra recorded on $\mathrm{Rh}(\mathrm{CO})_{2}(3,6-\mathrm{DBSQ})$ in hexane solution show a marked dependence on temperature (figure 6). At room temperature, the band in the $1600 \mathrm{~nm}$ region that appeared in the solid-state spectrum is not observed. However, as the temperature of the solution is cooled to the point where crystals of the complex form and separate from solution, the $1600 \mathrm{~nm}$ transition grows in intensity. Stacked aggregates of complex molecules form in solution with decreasing temperature. It may be concluded that the $1600 \mathrm{~nm}$ transition is a property of the molecular assembly, rather than an intrinsic property of an individual complex molecule. The nature of this transition is relevant to understanding the photomechanical effect. It may be associated with the band structure formed by interacting $p_{z}$ and $d_{z}{ }^{2}$ metal orbitals along the axial length of the

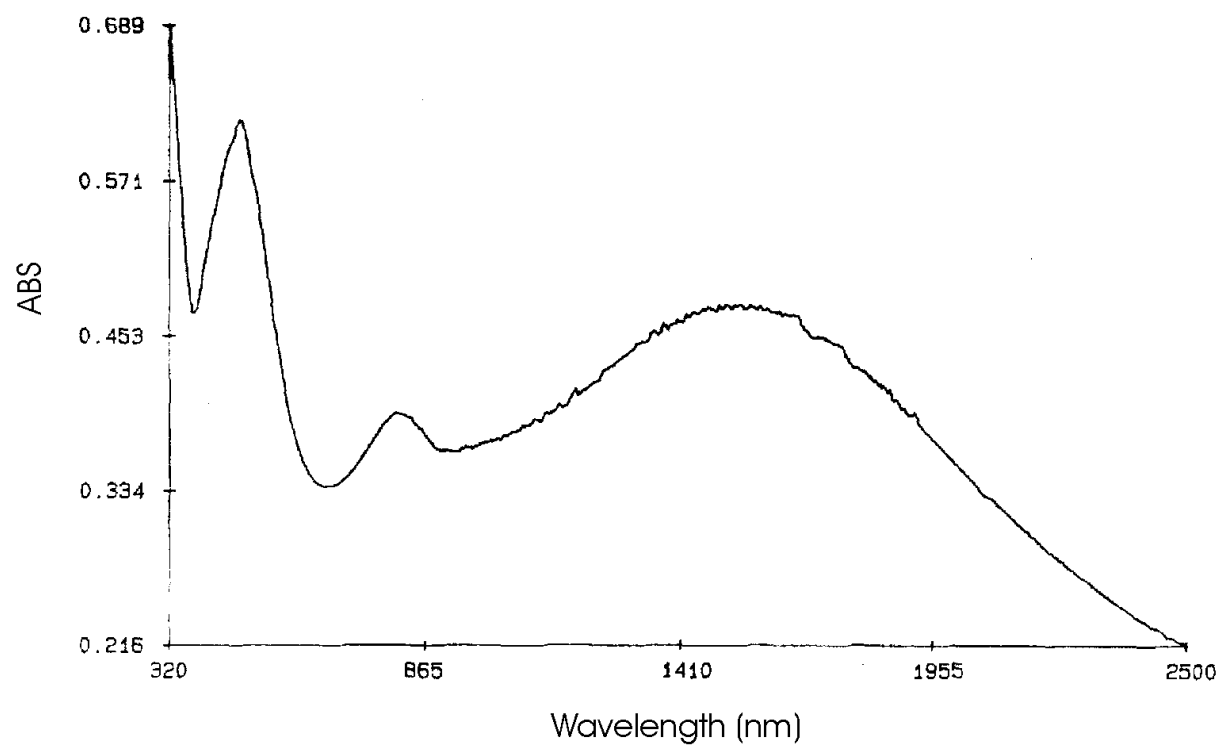

Figure 3. The optical spectrum of $\mathrm{Rh}(\mathrm{CO})_{2}(3,6-\mathrm{DBSQ})$ recorded in the solid state as a $\mathrm{KBr}$ pellet. 


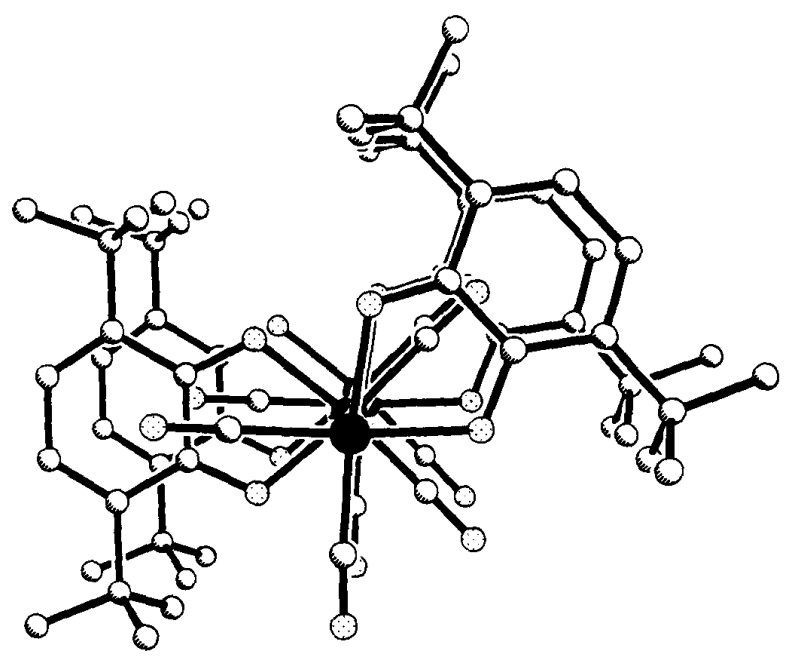

Figure 4. A view down the axis of stacked $\mathrm{Rh}(\mathrm{CO})_{2}(3,6-\mathrm{DBSQ})$ molecules in the solid state.

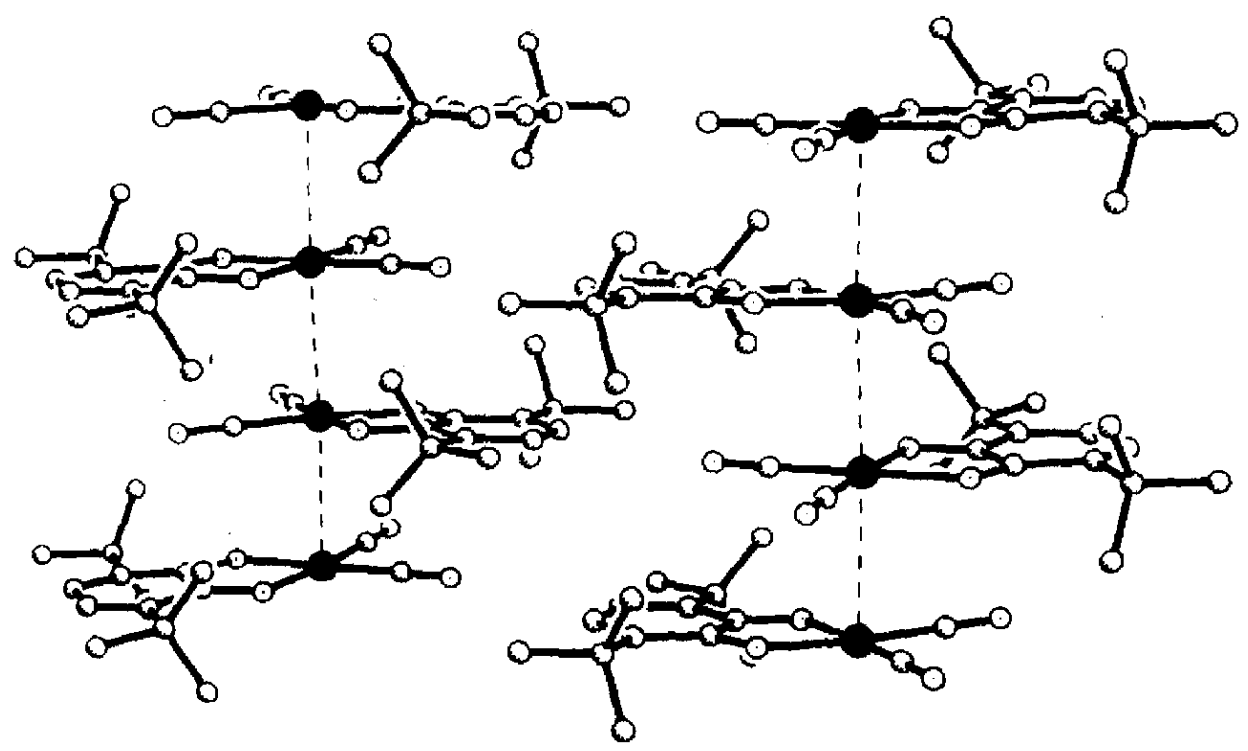

Figure 5. A view of the one-dimensional stacks of $\mathrm{Rh}(\mathrm{CO})_{2}(3,6-\mathrm{DBSQ})$ molecules that form in the solid state.

stack. Low energy transitions of this type have been reported for stacked, planar Pt(II) complexes and are observed to be polarized along the axial length of the solid state lattice $^{6}$. An alternative assignment for this transition of $\mathrm{Rh}(\mathrm{CO})_{2}(3,6-\mathrm{DBSQ})$ is as a metal-ligand charge-transfer band from the filled antibonding level resulting from axial $d_{z}{ }^{2}$ orbital interactions to the vacant semiquinonate $\pi^{*}$ orbital. The transfer of charge from 
the core antibonding orbital would strengthen interactions between metals along the stack with a slight contraction in $\mathrm{Rh}-\mathrm{Rh}$ separations. This contraction on the irradiated side of the linear crystal would result in the mechanical response (scheme 1). A decrease of $0 \cdot 2 \AA$ in $\mathrm{Rh}-\mathrm{Rh}$ separation would result in a macroscopic decrease of approximately

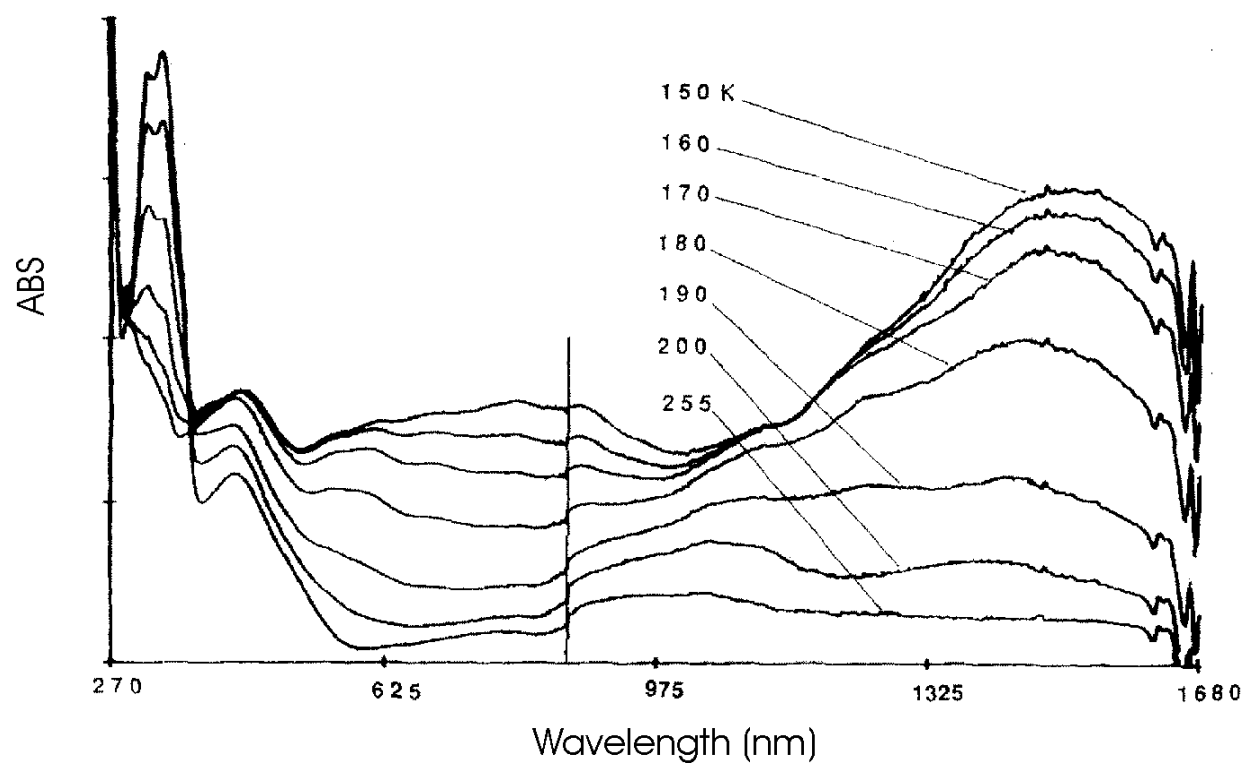

Figure 6. The temperature dependence of the electronic spectrum of $\mathrm{Rh}(\mathrm{CO})_{2}(3,6-$ DBSQ) in hexane solution.

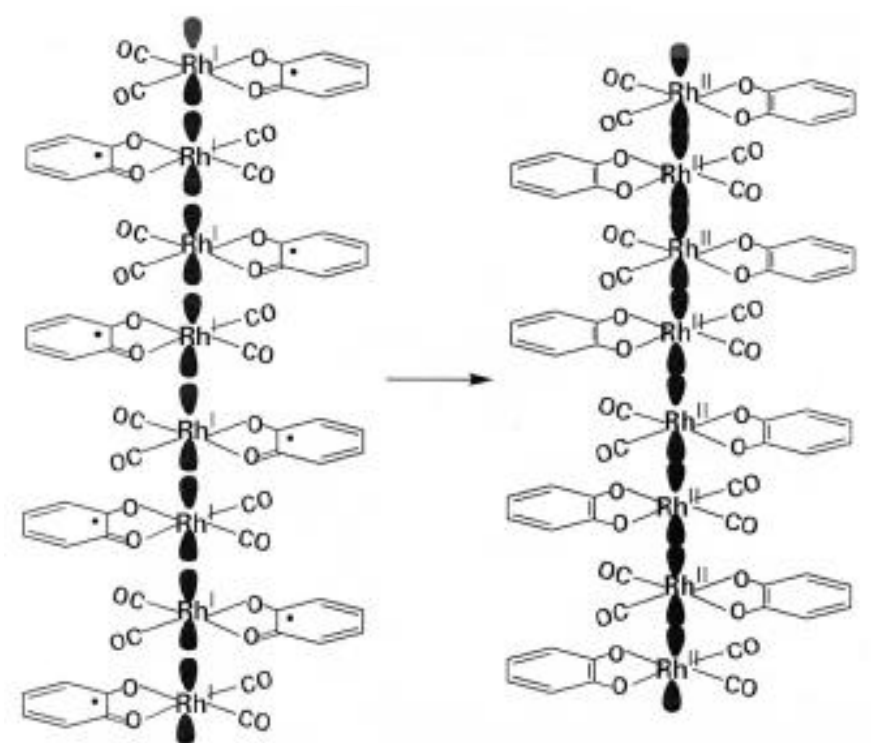

Scheme 1. 
$0.1 \mathrm{~mm}$ per millimeter of crystal length. In this case the transition would not exhibit axial polarization. Crystals of $\mathrm{Rh}(\mathrm{CO})_{2}(3,6-\mathrm{DBSQ})$ were attached to the surface of a piece of transparent adhesive tape with the assumption that needle alignment on the surface would be mainly edge-on rather than end-on. The observation of the $1600 \mathrm{~nm}$ transition is interpreted, from this relatively crude experiment, as an indication that the band is not axially polarized as a low-energy MLCT transition.

\section{Photomechanical property of polymeric $\left[\mathrm{Co}(\mu \text {-pyz) }(3,6-\mathrm{DBSQ})(3,6-\mathrm{DBCat})]_{n}\right.$}

Considerable interest has been directed to valence tautomerism (VT) in cobalt(III) complexes containing catecholate ligands. Optically induced shifts in charge distribution, with an accompanying spin transition for the metal ion, result in a relatively large changes in $\mathrm{Co}-\mathrm{L}$ bond lengths as the metal shifts between ls-Co(III) and hs-Co(II). These effects have been described with more detail in a recent review ${ }^{4}$. The class of complexes that has been of greatest interest in studies on VT contain mixed charge semiquinonate and catecholate ligands coordinated to $\mathrm{Co}$ (III) in an octahedral complex also containing ancillary nitrogen-donor ligands, $\mathrm{Co}^{\mathrm{III}}(\mathrm{N}-\mathrm{N})(3,6-\mathrm{DBSQ})(3,6-\mathrm{DBCat})$. An intense optical transition in the $2500 \mathrm{~nm}$ region is typically observed for these complexes. DFT calculations indicate that the band is a Cat $\rightarrow$ SQ LL'CT transition. However, the energy

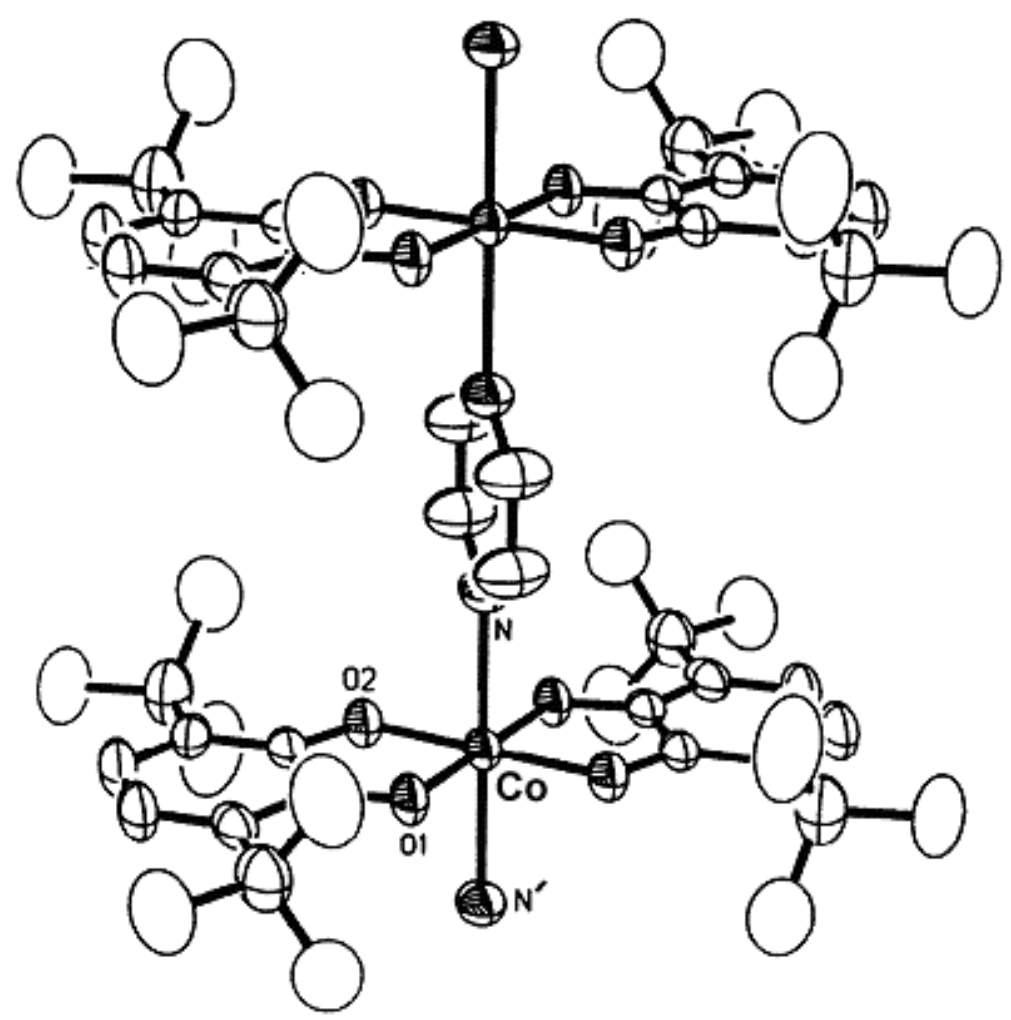

Figure 7. A view showing one unit of the $[\mathrm{Co}(\mu \text {-pyz)(3,6-DBSQ)(3,6-DBCat })]_{n}$ polymer. 
of the transition is close to the energy required to induce a shift in charge distribution to the $\mathrm{Co}^{\mathrm{II}}(\mathrm{N}-\mathrm{N})(3,6-\mathrm{DBSQ})_{2}$ redox isomer containing hs-Co(II). In a linear polymer the optically induced shift in charge and spin state of the Co ion would produce a photomechanical effect similar to that of $\mathrm{Rh}(\mathrm{CO})_{2}(3,6-\mathrm{DBSQ})$.

The reaction carried out by first treating $\mathrm{Co}_{2}(\mathrm{CO})_{8}$ with 3,6-di-tert-butyl-1,2-benzoquinone, followed by addition of pyrazine, in hexane/THF solution gave crystals of the $\left[\mathrm{Co}^{\text {IIII }}(\mu \text { pyz) }(3,6-\mathrm{DBSQ})(3,6-\mathrm{DBCat})]_{n}\right.$ polymer ${ }^{7}$. Tetragonal crystals of the polymer form as thin plates with molecules of hexane occluded in the lattice between adjacent polymer strands. Views of a repeating unit of the polymer and the packing arrangement are shown in figures 7 and 8. The hexane molecules of crystallization are slowly displaced from the lattice at room temperature. Consequently, crystallographic analysis and photomechanical experiments were carried out at low temperature. Structural features of the Co complex indicate that the metal ion is in the 1s-Co(III) state with mixed charge SQ and Cat ligands. A broad, intense LL'CT transition is observed for the complex in the solid state at $2500 \mathrm{~nm}^{7}$. Changes in the intensity of this band with increasing temperature, with temperature-dependent changes in magnetic moment indicate that there is a thermally-induced shift in charge distribution to the Co(II) redox isomer at temperatures above room temperature. Photomechanical experiments were carried out using the experimental arrangement described earlier using a tungsten lamp as the incident light source and a cold $\mathrm{N}_{2}$ stream to suppress crystal deterioration. The light response was observed as a slight curling of the corners of the thin tetragonal plate. This

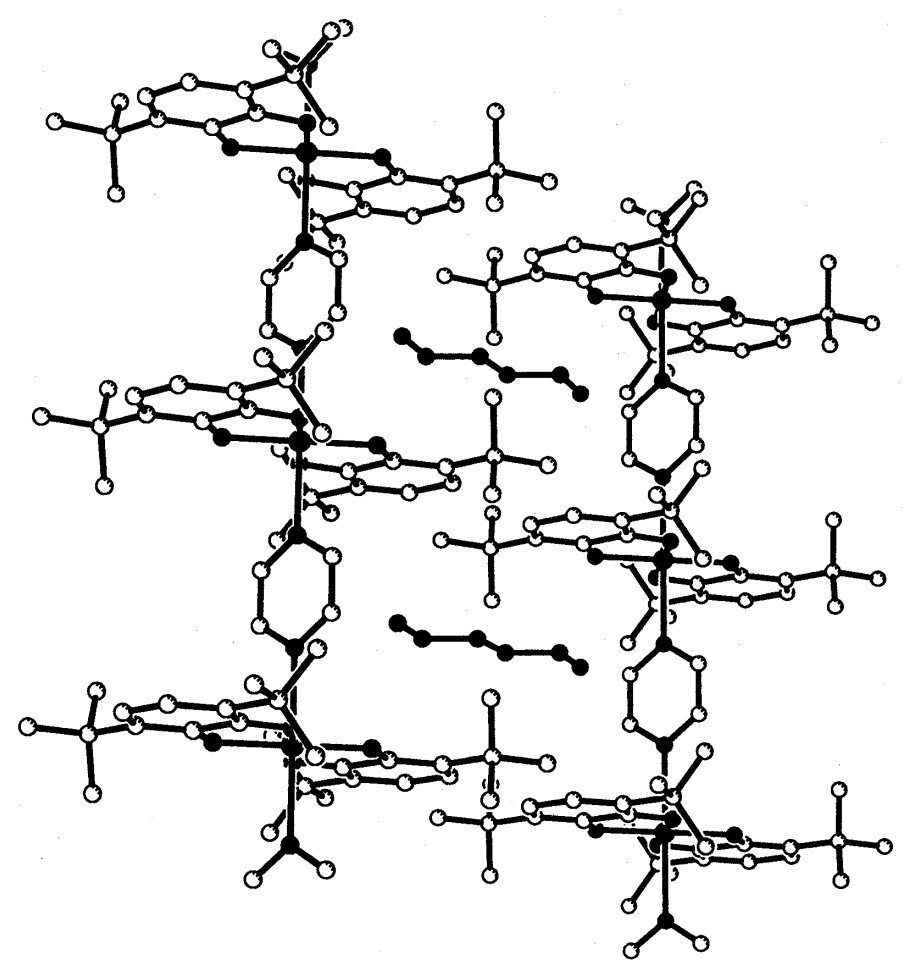

Figure 8. The crystal packing diagram of the [Co( $\mu$-pyz)(3,6-DBSQ)(3,6-DBCat) $]_{n}$ polymer showing the occluded molecules of hexane. 
effect is far less dramatic than the reversible bending response of $\mathrm{Rh}(\mathrm{CO})_{2}(3,6-\mathrm{DBSQ})$, but it demonstrates the effect of the light-induced change in polymer length on the irradiated side of the plate.

\section{Conclusions}

Transition metal complexes containing radical semiquinonate ligands often show intense low energy MLCT transitions that are associated with a shift in charge distribution within the complex. In cases where complex molecules align in the solid state to form a stacked structure, and the optically-induced shift in charge distribution results in a physical change in the separation between units of the stack, crystals may exhibit photomechanical properties. The crystal bending effect appears to result from an anisotropic change in length of the stack axis on the irradiated side of the crystal. Tert-butyl substituents on the ring of quinone ligands appear to give a 'soft' solid state lattice with special separation between molecular stacks that contributes to a reversible bending effect, rather than crystal fracture upon irradiation.

\section{Acknowledgement}

This research was supported by the National Science Foundation.

\section{References}

1. Pierpont C G and Buchanan R M 1981 Coord. Chem. Rev. 3845

2. Pierpont C G and Lange C W 1994 Prog. Inorg. Chem. 41331

3. Pierpont C G 2001 Coord. Chem. Rev. 219-221 415

4. Pierpont C G 2001 Coord. Chem. Rev. 216-217 99

5. Lange C W, Foldeaki M, Nevodchikov V I, Cherkasov V K, Abakumov G A and Pierpont CG 1992 J. Am. Chem. Soc. 1144220

6. Lewis N S, Mann K R, Gordon J G II and Gray H B 1976 J. Am. Chem. Soc. 987461

7. Jung O-S and Pierpont C G 1994 J. Am. Chem. Soc. 1162229 